\title{
GENERALIZED HARMONIC NUMBER IDENTITIES AND A RELATED MATRIX REPRESENTATION
}

\author{
Gi-Sang Cheon and Moawwad E. A. El-Mikkawy
}

\begin{abstract}
In this paper, we obtain important combinatorial identities of generalized harmonic numbers using symmetric polynomials. We also obtain the matrix representation for the generalized harmonic numbers whose inverse matrix can be computed recursively.
\end{abstract}

\section{Introduction and preliminaries}

The ordinary harmonic numbers are denoted by $H_{n}$ and are defined as

$$
H_{0}=0 \quad \text { and } \quad H_{n}=\sum_{k=1}^{n} \frac{1}{k}, \quad n=1,2, \ldots
$$

The first few harmonic numbers are $1, \frac{3}{2}, \frac{11}{6}, \frac{25}{12}, \frac{137}{60}, \ldots$ These harmonic numbers were studied in antiquity and are important in various branches of number theory and combinatorial problems. They are closely related to the Riemann zeta function defined by

$$
\zeta(s)=\sum_{n=1}^{\infty} \frac{1}{n^{s}}=\prod_{p}\left(1-p^{-s}\right)^{-1},
$$

where the product is over all primes $p$, and appear in various expressions for various special functions. It is well known that

$$
H_{n}=\frac{|s(n+1,2)|}{n !},
$$

where $s(n, k)$ denotes the Stirling numbers of the first kind defined by

$$
(x)_{n}:=\prod_{r=1}^{n}(x-r+1)=\sum_{k=0}^{n} s(n, k) x^{k} .
$$

For a notational convenience, we denote $c(n, k)=|s(n, k)|$, i.e., $c(n, k)$ is the unsigned Stirling number of the first kind which counts the permutations of $n$

Received June 1, 2006.

2000 Mathematics Subject Classification. 05A30.

Key words and phrases. harmonic numbers, Riemann zeta function, Stirling numbers, Bernoulli numbers, symmetric polynomials. 
elements that are the product of $k$ disjoint cycles. Further, It is known [9] that

$$
\zeta(k+1)=\sum_{n=k}^{\infty} \frac{c(n, k)}{n \cdot n !} .
$$

In many recent works (see for example [1]-[5],[7]-[10]), the harmonic numbers have been generalized by several ways and the related identities were obtained. Our observations suggest that the generalized harmonic numbers can be viewed combinatorially.

For instance, the generalized harmonic numbers $H_{n}^{(r)}$ of order $r$ are defined to be partial sums of the Riemann zeta function:

$$
H_{0}^{(r)}=0 \quad \text { and } \quad H_{n}^{(r)}=\sum_{k=1}^{n} \frac{1}{k^{r}}, \quad n, r \geq 1 .
$$

It is known (p.217 in [6]) that the numbers $H_{n}^{(r)}$ and $c(n, r)$ are connected by

$$
\begin{aligned}
c(n+1,1) & =n ! \\
c(n+1,2) & =n ! H_{n}, \\
c(n+1,3) & =\frac{n !}{2}\left(H_{n}^{2}-H_{n}^{(2)}\right), \\
c(n+1,4) & =\frac{n !}{6}\left(H_{n}^{3}-3 H_{n} H_{n}^{(2)}+2 H_{n}^{(3)}\right),
\end{aligned}
$$

and so on. In [1], Adamchik obtained the general formula for $c(n, m)$ in terms of generalized harmonic numbers $H_{n}^{(r)}$ :

$$
c(n, m)=\frac{(n-1) !}{(m-1) !} w(n, m-1)
$$

where the $w$-sequence is defined recursively by

(4) $\quad w(n, 0)=1, \quad w(n, m)=\sum_{k=0}^{m-1}(-1)^{k}(m-1)_{k} H_{n-1}^{(k+1)} w(n, m-1-k)$.

And he showed the $w$-sequence can be rewritten through a multiple sum:

$$
w(n, m)=\sum_{i_{1}=1}^{n-1} \sum_{i_{2}=i_{1}+1}^{n-1} \ldots \sum_{i_{m}=i_{m-1}+1}^{n-1} \frac{m !}{i_{1} i_{2} \cdots i_{m}} .
$$

In [5], Chu and Donno defined the generalized harmonic numbers $H_{n}(r)$ by

$$
H_{0}(r)=0 \quad \text { and } \quad H_{n}(r)=\sum_{k=1}^{n} \frac{1}{k+r}, \quad n \geq 1,
$$

and they obtained several striking identities on the ordinary harmonic numbers. 
For the rising factorial $[x]_{n}=x(x+1) \cdots(x+n-1)(n \geq 1)$, by writing $\frac{1}{[r]_{p+1}}$ as the sum of $p+1$ partial fractions we obtain:

$$
\frac{1}{[r]_{p+1}}=\sum_{k=0}^{p} \frac{A_{k}^{(p)}}{k+r}=\frac{1}{p !} \sum_{k=0}^{p}\left(\begin{array}{l}
p \\
k
\end{array}\right) \frac{(-1)^{k}}{k+r},
$$

where $A_{k}^{(p)}=1 / \prod_{t=0}^{p}(t-k), t \neq k$. It is easy to show that (6) can also be obtained by using the fact that

$$
\Delta^{n}\left(\frac{1}{x}\right)=(E-1)^{n}\left(\frac{1}{x}\right)=(-1)^{n} \frac{n !}{[x]_{n+1}},
$$

where $\Delta$ and $E$ are the forward and the shift operators with unit step, respectively. The rising factorial $[r]_{p+1}$ satisfies the following identity, typically proved by induction or telescoping sums [11]:

$$
\sum_{r=1}^{n} \frac{1}{[r]_{p+1}}=\frac{\left(\begin{array}{c}
n+p \\
p
\end{array}\right)-1}{p(p !)\left(\begin{array}{c}
n+p \\
p
\end{array}\right)}, \quad p \geq 1 .
$$

Using (6) and (7), we can establish an interesting identity for the generalized harmonic numbers $H_{n}(r)$ :

$$
\sum_{r=0}^{p}(-1)^{r}\left(\begin{array}{l}
p \\
r
\end{array}\right) H_{n}(r)=\frac{\left(\begin{array}{c}
n+p \\
p
\end{array}\right)-1}{p\left(\begin{array}{c}
n+p \\
p
\end{array}\right)} .
$$

Besides, in [2], Benjamin et. al. showed that the generalized harmonic numbers $H_{n}^{<r>}$ defined by

$$
H_{n}^{<0>}=\frac{1}{n} \quad \text { and } \quad H_{n}^{<r>}=\sum_{r=1}^{n} H_{k}^{<r-1>}, \quad n, r \geq 1
$$

can be expressed in terms of $r$-Stirling numbers. In 1997, Santmyer [10] defined the generalized harmonic numbers $H_{n, r}$ of rank $r$ by

$$
H_{n, r}=\sum_{k_{0}+\cdots+k_{r} \leq n} \frac{1}{k_{0} k_{1} \cdots k_{r}}, \quad n \geq 1, r \geq 0 .
$$

Note that these generalized harmonic numbers $H_{n}^{(r)}, H_{n}(r), H_{n}^{<r>}$ and $H_{n, r}$ reduce to the ordinary harmonic numbers $H_{n}$ when $r=1$ or $r=0$.

In this paper, we define other generalized harmonic numbers

$$
H(n, r):=\frac{c(n+1, r+1)}{n !}
$$

which are direct generalization of (1). The purpose of this paper is to obtain some interesting identities involving $H(n, r)$. These results are derived from symmetric polynomials. Further, we give a matrix representation for $H(n, r)$. 


\section{Symmetric polynomials}

In this section, we are going to consider the so-called symmetric polynomials which are not only important tool in mathematics but also play considerable roles in computer science, physics and statistics.

A polynomial $P\left(x_{1}, x_{2}, \ldots, x_{n}\right)$ in the variables $x_{1}, x_{2}, \ldots, x_{n}$ is called a symmetric polynomial or symmetric function if it is invariant under all possible permutations of the variables $x_{1}, x_{2}, \ldots, x_{n}$. Especially, important symmetric polynomials that will be considered in the current paper are the elementary symmetric polynomial $\sigma_{k}^{(n)}$, the complete symmetric polynomial $\tau_{k}^{(n)}$, and the power sum symmetric polynomial $S_{k}^{(n)}$ on the variables $x_{1}, x_{2}, \ldots, x_{n}$. These polynomials for integers $n, k$ with $n \geq k \geq 0$ are defined by:

$$
\begin{aligned}
& \text { - } \sigma_{k}^{(n)}\left(x_{1}, x_{2}, \ldots, x_{n}\right)=\sum_{1 \leq r_{1}<\cdots<r_{k} \leq n} x_{r_{1}} x_{r_{2}} \cdots x_{r_{k}}, \\
& \text { - } \tau_{k}^{(n)}\left(x_{1}, x_{2}, \ldots, x_{n}\right)=\sum_{1 \leq r_{1} \leq \cdots \leq r_{k} \leq n} x_{r_{1}} x_{r_{2}} \cdots x_{r_{k}}, \\
& \text { - } S_{k}^{(n)}\left(x_{1}, x_{2}, \ldots, x_{n}\right)=\sum_{r=1}^{n} x_{r}^{k},
\end{aligned}
$$

where $\sigma_{0}^{(n)}\left(x_{1}, x_{2}, \ldots, x_{n}\right)=1$ and $\tau_{0}^{(n)}\left(x_{1}, x_{2}, \ldots, x_{n}\right)=1$.

Lemma 1. (Newton-Girard identity [13]) For positive integers $m, n$ such that $1 \leq m \leq n$, the following holds:

$$
\begin{gathered}
m \sigma_{m}^{(n)}\left(x_{1}, \ldots, x_{n}\right) \\
=\sum_{r=1}^{m}(-1)^{r+1} S_{r}^{(n)}\left(x_{1}, \ldots, x_{n}\right) \sigma_{m-r}^{(n)}\left(x_{1}, \ldots, x_{n}\right) .
\end{gathered}
$$

It is worthy to mention that two polynomials $\sigma_{k}^{(n)}$ and $\tau_{k}^{(n)}$ can be written through multiple sums as follow:

$$
\begin{aligned}
\sigma_{k}^{(n)}\left(x_{1}, x_{2}, \ldots, x_{n}\right) & =\sum_{r_{k}=k}^{n} \sum_{r_{k-1}=k-1}^{r_{k}-1} \cdots \sum_{r_{1}=1}^{r_{2}-1} x_{r_{1}} x_{r_{2}} \cdots x_{r_{k}}, \\
\tau_{k}^{(n)}\left(x_{1}, x_{2}, \ldots, x_{n}\right) & =\sum_{r_{1}=1}^{n} \sum_{r_{2}=r_{1}}^{n} \cdots \sum_{r_{k}=r_{k-1}}^{n} x_{r_{1}} x_{r_{2}} \cdots x_{r_{k}} .
\end{aligned}
$$

It is known that two generating functions $E(t)$ and $F(t)$ for $\sigma_{k}^{(n)}$ and $\tau_{k}^{(n)}$ are given respectively by

$$
\begin{aligned}
& E(t)=\prod_{i=1}^{n}\left(1+x_{i} t\right)=\sum_{r=0}^{n} \sigma_{r}^{(n)}\left(x_{1}, x_{2}, \ldots, x_{n}\right) t^{r} \\
& F(t)=\prod_{i=1}^{n} \frac{1}{1-x_{i} t}=\sum_{r=0}^{\infty} \tau_{k}^{(n)}\left(x_{1}, x_{2}, \ldots, x_{n}\right) t^{r}
\end{aligned}
$$


These functions satisfy $E(t) F(-t)=F(t) E(-t)=1$ and

$$
\sum_{m=0}^{r}(-1)^{m} \sigma_{m}^{(r)} \tau_{r-m}^{(r)}=\delta_{r 0}
$$

for specific non-negative integers $n$ and $r$ such that $0 \leq r \leq n$, where $\delta_{n k}$ is the Kronecker symbol. The unsigned Stirling numbers of the first kind $c(n, k)$ and the Stirling numbers of the second kind $S(n, k)$ defined by $x^{n}=$ $\sum_{k=0}^{n} S(n, k)(x)_{k}$ are related to $\sigma_{k}^{(n)}$ and $\tau_{k}^{(n)}$ by

$$
\begin{aligned}
c(n, k) & =\sigma_{n-k}^{(n-1)}(1,2, \ldots, n-1), \\
S(n, k) & =\tau_{n-k}^{(k)}(1,2, \ldots, k) .
\end{aligned}
$$

Before we consider more generalized harmonic number identities it may be useful to give the following result.

Lemma 2. For all nonzero real numbers $x_{1}, x_{2}, \ldots, x_{n}$ and for all nonnegative integer $i$, we have

$$
\sigma_{i}^{(n)}\left(\frac{1}{x_{1}}, \frac{1}{x_{2}}, \ldots, \frac{1}{x_{n}}\right)=\frac{1}{x_{1} x_{2} \cdots x_{n}} \sigma_{n-i}^{(n)}\left(x_{1}, x_{2}, \ldots, x_{n}\right) .
$$

Proof. Replacing each $x_{i}$ by $\frac{1}{x_{i}}$ in (16) gives:

$$
\sum_{r=0}^{n} \sigma_{r}^{(n)}\left(\frac{1}{x_{1}}, \frac{1}{x_{2}}, \ldots, \frac{1}{x_{n}}\right) t^{r}=\frac{1}{x_{1} x_{2} \cdots x_{n}} \prod_{i=1}^{n}\left(t+x_{i}\right)
$$

Hence

$$
\sum_{i=0}^{n} \sigma_{i}^{(n)}\left(\frac{1}{x_{1}}, \frac{1}{x_{2}}, \ldots, \frac{1}{x_{n}}\right) t^{i}=\frac{1}{x_{1} x_{2} \cdots x_{n}} \sum_{i=0}^{n} \sigma_{n-i}^{(n)}\left(x_{1}, x_{2}, \ldots, x_{n}\right) t^{i} .
$$

The result follows.

As a direct consequence of Lemma 2, we see that for specific positive integer $n$, the special case $x_{i}=i$ for each $i=1,2, \ldots, n$ yields:

$$
\sigma_{i}^{(n)}\left(1, \frac{1}{2}, \ldots, \frac{1}{n}\right)=\frac{1}{n !} \sigma_{n-i}^{(n)}(1,2, \ldots, n) .
$$

Lemma 3. For integers $n, m$ with $n \geq m \geq 2$, we have

$$
c(n, m)=(n-1) ! \sigma_{m-1}^{(n-1)}\left(1, \frac{1}{2}, \ldots, \frac{1}{n-1}\right) .
$$

Proof. With the help of (22) and (19) we obtain

$$
\frac{c(n, m)}{(n-1) !}=\frac{1}{(n-1) !} \sigma_{n-m}^{(n-1)}(1,2, \ldots, n-1)=\sigma_{m-1}^{(n-1)}\left(1, \frac{1}{2}, \ldots, \frac{1}{n-1}\right),
$$

which proves $(23)$. 
From Lemma 3 and (3), we immediately obtain a simple representation for the $w$-sequence:

$$
w(n, m)=m ! \sigma_{m}^{(n-1)}\left(1, \frac{1}{2}, \ldots, \frac{1}{n-1}\right) .
$$

Further, using the recurrence relation (25) enables us to compute the numbers $w(n, w)$ recursively as follows:

$$
w(i, j)=w(i-1, j)+\frac{j}{i-1} w(i-1, j-1), \quad i \geq 2, j \geq 1
$$

so that $w(i, i)=0$ and $w(i, 0)=1$ for $i \geq 0$, and $w(i, 1)=H_{i-1}$ for $i \geq 1$.

\section{Generalized harmonic numbers $H(n, r)$}

We begin with the recurrence relation for the unsigned Stirling numbers of the first kind $c(n, r)$ in [6]:

$$
c(n, r)=c(n-1, r-1)+(n-1) c(n-1, r), \quad n, r \geq 1
$$

with $c(n, 0)=\delta_{n 0}, c(n, n)=1, c(n, 1)=(n-1)$ ! for $n \geq 1$. The relation (25) also gives $c(n+1,2)=c(n, 1)+n c(n, 2)$. Dividing both sides by $n$ ! taking into account the fact that $c(n, 1)=(n-1)$ !, we obtain

$$
g(n)=\frac{1}{n}+g(n-1), \quad n \geq 1,
$$

where $g(n)=c(n+1,2) / n$ !. Since $H_{n}=\frac{1}{n}+H_{n-1}, n \geq 1$, from (26) we obtain $g(n)=H_{n}$ which proves (1).

More generally, we may rewrite the recurrence relation (25) in the form:

$$
\frac{c(n+1, r+1)}{n !}=\frac{1}{n} \frac{c(n, r)}{(n-1) !}+\frac{c(n, r+1)}{(n-1) !} .
$$

Let us define the generalized harmonic numbers $H(n, r)$ by

$$
H(n, 0)=1 \quad \text { and } H(n, r)=\frac{c(n+1, r+1)}{n !} \quad n, r \geq 1 .
$$

First note that from (27) the numbers $H(n, r)$ satisfy the recurrence relation:

$$
H(n, r)=H(n-1, r)+\frac{1}{n} H(n-1, r-1) .
$$

Theorem 4. The generalized harmonic numbers $H(n, r)$ satisfy

$$
H(n, r)=\sum_{1 \leq k_{1}<\cdots<k_{r} \leq n} \frac{1}{k_{1} k_{2} \cdots k_{r}} .
$$

Proof. From Lemma 3, we have

$$
H(n, r)=\sigma_{r}^{(n)}\left(1, \frac{1}{2}, \ldots, \frac{1}{n}\right) .
$$


Hence using (10) yields

$$
H(n, r)=\sum_{1 \leq k_{1}<\cdots<k_{r} \leq n} \frac{1}{k_{1} k_{2} \cdots k_{r}} .
$$

This completes the proof.

The formula (30) can be used to find a formula for coefficients of powers of $m$ in the Stirling numbers $S(m+n, m)$ of the second kind (see [8]).

At this stage it is convenient to obtain a generating function for the generalized harmonic numbers $H(n, r)$. From (16), we obtain

$$
\sum_{r=0}^{n} \sigma_{n-r}^{(n)}\left(x_{1}, x_{2}, \ldots, x_{n}\right) t^{r}=\prod_{i=1}^{n}\left(t+x_{i}\right) .
$$

Setting $x_{m}=m$ for each $m=1,2, \ldots, n$ in (31) gives

$$
\sum_{r=0}^{n} \sigma_{n-r}^{(n)}(1,2, \ldots, n) t^{r}=\prod_{i=1}^{n}(t+i)
$$

Thus from (19) we have

$$
\sum_{r=0}^{n} c(n+1, r+1) t^{r}=\prod_{i=1}^{n}(t+i) .
$$

Using (28) together with $(x)_{n}=n !\left(\begin{array}{l}x \\ n\end{array}\right)$ and (32) proves that the generating function we are looking for is given by

$$
\sum_{r=0}^{n} H(n, r) t^{r}=\left(\begin{array}{c}
n+t \\
n
\end{array}\right)
$$

Since $H(n, r)=\frac{1}{r !} w(n+1, r)$, the generating function for $w(n, k)$ in $(3)$ can be easily obtained.

The Bernoulli polynomials $B_{n}(x)$ defined by

$$
B_{n}(x)=\sum_{k=0}^{n}\left(\begin{array}{l}
n \\
k
\end{array}\right) B_{n-k} x^{k}
$$

are important in obtaining closed form expressions for sum of powers of integers [11] such as

$$
\sum_{r=0}^{n} r^{k}=\frac{1}{k+1}\left(B_{k+1}(n+1)-B_{k+1}\right), \quad k \geq 0,
$$

where $B_{n}=B_{n}(0)$ is the $n$-th Bernoulli number.

Theorem 5. The generalized harmonic numbers $H(n, r)$ satisfy the following identities:

(i) $\sum_{r=1}^{n} H(n, r)=n$,

(ii) $\sum_{r=1}^{n=1}(-1)^{r+1} H(n, r)=1$, 
(iii) $\sum_{j=r}^{n} \frac{1}{j} H(j-1, r-1)=H(n, r)$,

(iv) $\sum_{r=0}^{n}(-1)^{r} H(n, r)\left(B_{r+1}(n+1)-B_{r+1}\right) \frac{1}{r+1}=1$,

(v) $\sum_{r=0}^{n} H(n, r)\left(B_{r+1}(n+1)-B_{r+1}\right) \frac{1}{r+1}=\left(\begin{array}{c}2 n+1 \\ n\end{array}\right)$,

(vi) $\sum_{r=1}^{n} r H(n, r)=(n+1)\left(H_{n+1}-1\right)$,

(vii) $\sum_{r=0}^{n}\left(2^{r}-1\right) H(n, r)=\left(\begin{array}{c}n+1 \\ 2\end{array}\right)$.

Proof. Putting $t=1$ in (33) and taking into account the fact that $H(n, 0)=1$ gives (i). Similarly putting $t=-1$ in (33) yields the identity (ii). To prove (iii), since

$$
H(n, r)=\sigma_{r}^{(n)}\left(1, \frac{1}{2}, \ldots, \frac{1}{n}\right)=\sum_{1 \leq k_{1}<\cdots<k_{r} \leq n} \frac{1}{k_{1} k_{2} \cdots k_{r}},
$$

applying (14) gives

$$
\begin{aligned}
H(n, r)= & \sum_{k_{r}=r}^{n} \sum_{k_{r-1}=r-1}^{k_{r}-1} \cdots \sum_{k_{1}=1}^{k_{2}-1} \frac{1}{k_{1} k_{2} \cdots k_{r}} \\
= & \frac{1}{r} \sum_{k_{r-1}=r-1}^{r-1} \sum_{k_{r-2}=r-2}^{k_{r-1}-1} \cdots \sum_{k_{1}=1}^{k_{2}-1} \frac{1}{k_{1} k_{2} \cdots k_{r-1}} \\
& +\frac{1}{r+1} \sum_{k_{r-1}=r-1}^{r} \sum_{k_{r-2}=r-2}^{k_{r-1}-1} \cdots \sum_{k_{1}=1}^{k_{2}-1} \frac{1}{k_{1} k_{2} \cdots k_{r-1}}+\cdots \\
& +\frac{1}{n} \sum_{k_{r-1}=r-1}^{n-1} \sum_{k_{r-1}-1}^{k_{r-2}=r} \sum_{k_{1}=1}^{k_{2}-1} \frac{1}{k_{1} k_{2} \cdots k_{r-1}} \\
= & \frac{1}{r} H(r-1, r-1)+\frac{1}{r+1} H(r, r-1)+\cdots \\
& +\frac{1}{n} H(n-1, r-1) \\
= & \sum_{j=r}^{n} \frac{1}{j} H(j-1, r-1),
\end{aligned}
$$

as required. To prove (iv), putting $m=n$ and $x_{k}=k$ for each $k=1,2, \ldots, n$ in (13) gives

$$
n \sigma_{n}^{(n)}(1,2, \ldots, n)=\sum_{r=1}^{n}(-1)^{r+1} S_{r}^{(n)}(1,2, \ldots, n) \sigma_{n-r}^{(n)}(1,2, \ldots, n) .
$$

Hence we get

$$
\sum_{r=1}^{n}(-1)^{r+1} S_{r}^{(n)}(1,2, \ldots, n) \sigma_{n-r}^{(n)}(1,2, \ldots, n) \frac{1}{n !}=n .
$$


Consequently, by using (34) and (13) we obtain

$$
\sum_{r=1}^{n}(-1)^{r+1} H(n, r)\left(B_{r+1}(n+1)-B_{r+1}\right) \frac{1}{r+1}=n .
$$

The identity (35) can also be written in the form:

$$
\sum_{r=0}^{n}(-1)^{r} H(n, r)\left(B_{r+1}(n+1)-B_{r+1}\right) \frac{1}{r+1}=1 .
$$

To prove (v), from (33) we have

$$
\sum_{r=0}^{n} H(n, r) t^{r}=\left(\begin{array}{c}
n+t \\
n
\end{array}\right)=\frac{(n+t)_{n}}{n !}
$$

Hence

$$
\sum_{t=0}^{n} \sum_{r=0}^{n} H(n, r) t^{r}=\sum_{r=0}^{n} H(n, r)\left(\sum_{t=0}^{n} t^{r}\right)=\sum_{t=0}^{n}\left(\begin{array}{c}
n+t \\
n
\end{array}\right)=\left(\begin{array}{c}
2 n+1 \\
n
\end{array}\right) .
$$

Using (34) then (36) yields

$$
\sum_{r=0}^{n} H(n, r)\left(B_{r+1}(n+1)-B_{r+1}\right) \frac{1}{r+1}=\left(\begin{array}{c}
2 n+1 \\
n
\end{array}\right)
$$

which is the required result. To prove (vi), using (33) we obtain

$$
\sum_{r=0}^{n} H(n, r) t^{r}=\frac{(t+n)_{n}}{n !}=\frac{1}{n !} \prod_{r=1}^{n}(t+r) .
$$

Differentiating both sides of (37) with respect to $t$ gives

$$
\sum_{r=1}^{n} r H(n, r) t^{r-1}=\left(\begin{array}{c}
n+t \\
n
\end{array}\right) \sum_{r=1}^{n} \frac{1}{t+r}=\left(\begin{array}{c}
n+t \\
n
\end{array}\right)\left(H_{n+t}-H_{t}\right) .
$$

Putting $t=1$ in (38) yields

$$
\sum_{r=1}^{n} r H(n, r)=(n+1)\left(H_{n+1}-1\right)
$$

as required. To prove (vii), by using the forward operator $\Delta$ with unit step, then (37) yields

$$
\Delta\left(\sum_{r=0}^{n} H(n, r) t^{r}\right)=\Delta\left(\frac{(t+n)_{n}}{n !}\right)
$$

Hence

$$
\sum_{r=0}^{n} H(n, r) \Delta\left(t^{r}\right)=\frac{1}{n !} \Delta\left((t+n)_{n}\right)
$$


Consequently, we have

$$
\sum_{r=0}^{n} H(n, r)\left((t+1)^{r}-t^{r}\right)=\frac{n}{n !}(t+n)_{n-1}=\frac{(t+n)_{n-1}}{(n-1) !}=\left(\begin{array}{c}
n+t \\
n-1
\end{array}\right) .
$$

Setting $t=1$ in (39), we obtain

$$
\sum_{r=0}^{n}\left(2^{r}-1\right) H(n, r)=\left(\begin{array}{c}
n+1 \\
n-1
\end{array}\right)=\left(\begin{array}{c}
n+1 \\
2
\end{array}\right)
$$

This completes the proof of the theorem.

We list more new identities for the generalized harmonic numbers $H(n, r)$ without proofs.

(i) $H(n, r)=\frac{1}{n} \sum_{k=r}^{n}\left(\begin{array}{l}k \\ r\end{array}\right) H(n-1, k-1)$,

(ii) $\sum_{r=k}^{n} H(n, r) S(r+1, k+1)=\left(\begin{array}{l}n \\ k\end{array}\right) \frac{n+1}{(k+1) !}$,

(iii) $\sum_{k=0}^{n}(-1)^{k+1} H(n, k) B_{k+1}=\frac{n+1}{n+2}$,

(iv) $\sum_{k=1}^{n-r+1} \frac{H(n-k, r-1)}{k}=r H(n, r)$.

\section{Matrix representation}

For the generalized harmonic numbers $H(n, k)$ defined by $(28)$ we define the $n \times n$ matrix $H=\left[h_{i j}\right]_{1 \leq i, j \leq n}$ as follows:

$$
h_{i j}= \begin{cases}H(i, j) & \text { if } i \geq j, \\ 0 & \text { if } i<j .\end{cases}
$$

For example, the $5 \times 5$ matrix $H$ is given by

$$
H=\left[\begin{array}{ccccc}
1 & 0 & 0 & 0 & 0 \\
\frac{3}{2} & \frac{1}{2} & 0 & 0 & 0 \\
\frac{11}{6} & 1 & \frac{1}{6} & 0 & 0 \\
\frac{25}{12} & \frac{35}{24} & \frac{5}{12} & \frac{1}{24} & 0 \\
\frac{137}{60} & \frac{15}{8} & \frac{17}{24} & \frac{1}{8} & \frac{1}{120}
\end{array}\right] .
$$

Here the elements of the matrix $H$ are computed recursively using (29). Using (18) we see that the inverse matrix $Q=\left[q_{i j}\right]_{1 \leq i, j \leq}$ of $H$ is given by

$$
q_{i j}= \begin{cases}(-1)^{i+j} j ! \tau_{i-j}^{(j+1)}(1,2, \ldots, j+1) & \text { if } i \geq j \\ 0 & \text { if } i<j .\end{cases}
$$

Using (21) yields

$$
q_{i j}= \begin{cases}(-1)^{i+j} j ! S(i+1, j+1) & \text { if } i \geq j \\ 0 & \text { if } i<j\end{cases}
$$


For example, we obtain the $5 \times 5$ matrix $Q$ as follows:

$$
Q=\left[\begin{array}{ccccc}
1 & 0 & 0 & 0 & 0 \\
-3 & 2 & 0 & 0 & 0 \\
7 & -12 & 6 & 0 & 0 \\
-15 & 50 & -60 & 24 & 0 \\
31 & -180 & 390 & -360 & 120
\end{array}\right]
$$

It is worthy to mention that the elements of the inverse matrix $Q$ can also be computed recursively using the recurrence relation

$$
q_{i j}=j q_{i-1, j-1}-(j+1) q_{i-1, j}, \quad i=3,4, \ldots, n ; j=2,3, \ldots, i-1,
$$

with

$$
q_{i 1}=(-1)^{i+1}\left(2^{i}-1\right) \quad \text { for } \quad i \geq 1 ; \quad q_{i i}=i ! \quad \text { for } \quad i \geq 1 .
$$

Due to the identity (i) of Theorem 5 , the product $D H$ is a stochastic matrix, where $D=\operatorname{diag}\left(1, \frac{1}{2}, \ldots, \frac{1}{n}\right)$.

We conclude this paper by describing that the matrix representation for the generalized harmonic numbers may be used to get more identities and some combinatorial connections with other combinatorial numbers.

Acknowledgment. The authors would like to thank the referee for valuable comments and suggestions.

\section{References}

[1] V. Adamchik, On Stirling numbers and Euler sums, J. Comput. and Appl. Math. 79 (1997), no. 1, 119-130.

[2] A. T. Benjamin, D. Gaebler, and R. Gaebler, A combinatorial approach to hyperharmonic numbers, Integers (Elec. J. Combi. Number Theory) 3 (2003), A15, 1-9.

[3] S. Chaturvedi and V. Gupta, Identities involving elementary symmetric functions, J. Phys. A 33 (2000), no. 29, L251-L255.

[4] W. Chu, Harmonic number identities and Hermite-Padé approximations to the logarithm function, J. Approx. Theory 137 (2005), no. 1, 42-56.

[5] W. Chu and L. De Donno, Hypergeometric series and harmonic number identities, Adv. in Appl. Math. 34 (2005), no. 1, 123-137.

[6] L. Comtet, Advanced Combinatorics, D. Reidel Pub. Company, 1974.

[7] A. Gertsch, Generalized harmonic numbers, C. R. Acad. Sci. Paris, Sér. I Math. 324 (1997), no. 1, 7-10.

[8] I. M. Gessel, On Miki's identity for Bernoulli numbers, J. Number Theory 110 (2005), no. $1,75-82$.

[9] T. M. Rassias and H. M. Srivastava, Some classes of infinite series associated with the Riemann Zeta and Polygamma functions and generalized harmonic numbers, Appl. Math. Comput. 131 (2002), no. 2-3, 593-605.

[10] J. M. Santmyer, A Stirling like sequence of rational numbers, Discrete Math. 171 (1997), no. $1-3,229-235$.

[11] F. Scheid, Numerical Analysis, McGraw-Hill Book Company, 1968.

[12] A. J. Sommese, J. Verscheide, and C. W. Wampler, Symmetric functions applied to decomposing solution sets of polynomial systems, SIAM J. Numerical Analysis 40 (2002), no. $6,2026-2046$.

[13] http://mathworld.wolfram.com/Newton-GirardFormulas.html. 
Gi-SANG CheON

DEPARTMENT OF MATHEMATICS

SUNGKYUNKWAN UNIVERSITY

SUWON 440-746, KOREA

E-mail address: gscheonoskku.edu

MoaWWad E. A. El-MikKaWy

DEPARTMENT OF MATHEMATICS

FACULTY OF SCIENCE OF MANSOURA UNIVERSITY

MANSOURA 35516, EGYPT

E-mail address: mikkawy@yahoo.com 